\title{
Pedagogía resiliente para evitar la violencia en la escuela rural de cara a la salud mental de maestros y estudiantes ${ }^{1}$.
}

\author{
Ana Elvira Castañeda Cantillo ${ }^{2}$, German Rolando Vargas Rodríguez ${ }^{3 *}$

\begin{abstract}
El presente artículo, es producto de la investigación denominada: "Violencia y escuela en escenarios rurales" (fase 2): Alternativas pedagógicas para construir escenarios de paz; en el marco de la convocatoria interna de la Universidad Santo Tomás FODEIN 14 2019. Bogotá (Colombia). Bogotá (Colombia).
\end{abstract} \\ ${ }^{2}$ Docente investigadora Doctorado en Educación Universidad Santo Tomas. Psicóloga, Magister en psicología clínica y de familia; Doctora en Educación. \\ ${ }^{3}$ Docente investigador Maestría en Educación y Didáctica Universidad Santo Tomas. Licenciado en filosofía e Historia, Magister en historia y Doctor en \\ Educación. Bogotá (Colombia).
}

\section{RESUMEN}

Introducción. La violencia y el conflicto, han estado presentes en lo social, en lo político y en la vida cotidiana de los ciudadanos por generaciones enteras, pero lo que más preocupa es que ha permeado los contextos escolares en especial los rurales, afectando la salud mental de los miembros de la comunidad escolar. Dado que se genera temor, desesperanza y minusvalía para afrontar las situaciones adversas, surgiendo una incertidumbre relacional

Objetivos. Proponer alternativas pedagógicas resilientes en la escuela rural, para afrontar la violencia en sus diversas formas y así promover la construcción de escenarios de paz en pro de la salud mental de los maestros y estudiantes.

Método. se basó en un enfoque cualitativo, de carácter hermenéutico, el cual permitió identificar las creencias sobre violencia que tienen los maestros y los tipos de violencia que se presentan en la escuela rural de Córdoba, Colombia.

Resultados. Se encontró que la violencia escolar, se adopta como una alternativa entre los estudiantes, para hacer valer sus derechos y ganar respeto ante los miembros de la comunidad escolar; como una forma en que se ha naturalizado la violencia en la escuela, la familia, la comunidad y en general en el país.

Conclusiones. Es necesario implementar estrategias de intervención con base en la Pedagógica Resiliente, para trasformar las creencias sobre violencia que se presentan en la escuela, dado el aumento en frecuencia y grado de agresividad en las diferentes formas de expresión de estas.

Palabras clave: Escuela rural, pedagogía Resiliente en salud mental.

\section{ABSTRACT \\ Resilient pedagogy to prevent violence in the rural school of face to the mental health of teachers and students.}

Introduction. Violence and conflict have been present in social, political and daily life of people for generations, it has permeated educational contexts as well, in special the rural ones, it concerns these communities to a great extent which deals to an ample effect in their mental health. Given that fear, hopelessness and handicap to face such unfavorable situations that lead to a relational incertitude.

Objectives. Propose resilient pedagogical alternatives in the rural educational environment, in order to confront the violence in its diverse manifestations, promoting the construction of peaceful scenarios in favour of teachers and students' mental health.

Method. Based on a qualitative hermeneutic approach, which allowed participants to identify the beliefs about violence, as well as the types of violence, that teachers and students in Cordoba's rural schools reckon.

Results. It was found out that school violence is adopted as an alternative between students as a means to gain respect among members of the school community; this represents a naturalization of violence in schools, families, communities and in the whole country itself.

Conclusions. It is necessary to implement strategies with foundations in the Resilient Pedagogy, with the aim to transform those beliefs of violence in schools, due to its growth in terms of frequency and aggressivity.

Keywords: Rural school, resilient pedagogy in mental health.

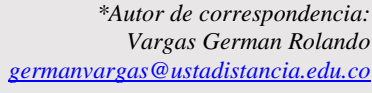

Como citar: Castañeda Cantillo A, Vargas Rodríguez G. Pedagogía resiliente para evitar la violencia en la escuela rural de cara a la salud mental de maestros y estudiantes ${ }^{l}$. Revista Cuarzo 2020;26(1):9-15.

Recibido: 13 de febrero de 2020 Aceptado: 28 de mayo de 2020 Publicado: 30 de junio de 2020

DOI: https://doi.org/10.26752/cuarzo.v26.n1.458 


\section{INTRODUCCIÓN}

$\mathrm{D}$ e acuerdo con la Comisión Económica para América Latina de las Naciones Unidas, la violencia está permeando todos los espacios sociales, ideas y creencias de aquellos que se encuentran en el ámbito educativo y especialmente el escolar, ya sea este urbano o rural (1). En la coyuntura actual colombiana y dentro de la apuesta humanista de la Universidad Santo Tomás, especialmente desde su Facultad de Educación, se busca contribuir a la comprensión y transformación de los factores y estructuras sociales que contribuyen al tratamiento del conflicto de forma violenta; de manera tal, que se puedan proponer alternativas pedagógicas resilientes (2), en aras de contribuir a salud mental de los maestros y a su vez les permita ofrecer a sus estudiantes el soporte requerido en escenarios violentos, para así evitar re victimizar a los sobrevivientes de la violencia.

Estar en escenarios permeados por el conflicto armado, genera en los diferentes miembros de la comunidad, afectación a la salud mental, la cual es definida por la Ley 1616 de 2003, como un proceso en permanente trasformación y movimiento presente en la cotidianidad del ser humano, evidenciándose en sus relaciones interpersonales, sus comportamientos "de manera tal que permite a los sujetos individuales y colectivos desplegar sus recursos emocionales, cognitivos y mentales para transitar por la vida cotidiana, para trabajar, para establecer relaciones significativas y para contribuir a la comunidad" ( 3 , p.15).

En este sentido la salud mental comprendida como la posibilidad en los seres humanos de percibir un equilibrio armónico entre las dimensiones emocionales, afectivas y sociales; viabiliza la construcción de estrategias para afrontar situaciones de diversa complejidad en la vida. Un Maestro que se percibe a sí mismo como competente para afrontar eventos de violencia en la escuela; podrá sobreponerse más fácil y prontamente, lo cual además de potenciar su dimensión personal; proveerá competencias profesionales que beneficiaran a sus estudiantes también.

Vargas y Castañeda (2), encontraron como tendencia que las acciones implementadas por los maestros y directivos en contextos rurales, frente a la violencia escolar; se basan en conocimiento empírico, construido en la experiencia misma, puesto que en sus carreras como licenciados; no se les formó al respecto, inclusive algunos maestros; vienen de disciplinas diferentes al campo especifico de la educación; por consiguiente carecen de capacitación especializada para atender población sobreviviente del conflicto armado Colombiano en condiciones de vulnerabilidad escolar en cualquiera de sus manifestaciones.

El objetivo de la investigación, fue proponer alternativas pedagógicas resilientes en la escuela rural, para afrontar la violencia en sus diversas formas y así promover la construcción de escenarios de paz en pro de la salud mental de los maestros y estudiantes como sobrevivientes. Bajo una perspectiva sistémica, se contemplan como parte de la pedagogía resiliente, aquellas acciones y reflexiones intencionadas del maestro por proveer escenarios dentro y fuera del aula, que apoyan la transición del estudiante de victima a sobreviviente ante situaciones de violencia, con herramientas para reconstruir su proyecto de vida, a partir de sus recursos y capacidades; de manera que se empodere como autor de otras formas de interacción en la escuela y con sus familias (5).

Para dar cuenta del objetivo se buscó identificar las creencias de los maestros sobre la violencia y sus formas de presentación en el entorno escolar; se abordó las creencias desde la perspectiva de (6) quien establece en estas la presencia de intenciones, argumentos y acciones, las cuales en su conjunto permiten dar un valor de verdad a las personas para afrontar situaciones de su vida.

Teniendo en cuenta que las escuelas deben considerarse como nicho de protección y cuidado del estudiante, dada su función central de formar para la vida. Se encuentra que cada día el fenómeno de la violencia escolar hace parte de la cotidianidad institucional, configurándose situaciones en las que prevalece la agresividad tanto física como verbal ya sea a nivel familiar, en las relaciones de maestros con estudiantes o entre iguales. Según la UNICEF (7), "se observa una tendencia creciente del acoso entre pares -agravado por el uso del internet-e inclusive de agresiones de estudiantes (sobre todo varones) y, en algunos casos, de padres contra maestros y autoridades educativas" ( 7 , p. 17).

\section{METODOLOGÍA}

Esta investigación se desarrolló bajo un enfoque cualitativo, en el cual, los investigadores, dada la complejidad del fenómeno abordado, estarán atentos a develar sus marcos de referencia con respecto a éste, para manejar ética y responsablemente la forma de interpretar las historias narradas por los participantes, las acciones, las intenciones, los hechos; evitando asignar juicios de valor a lo que emerja en el escenario investigativo. Ello conduce a que se confiere un valor determínate a las apreciaciones y formas de ver el mundo de los participantes.

López y Sandoval (8), al referirse, a los aportes de la perspectiva cualitativa en la investigación. Precisa que se genera un proceso "de carácter dialógico en el que las creencias, las mentalidades, los mitos, los prejuicios y los sentimientos, entre otros, son aceptados como elementos de análisis para producir conocimiento sobre la realidad humana." (8, p. 34). Dicho esto, el enfoque asumido, considera que los protagonistas de la investigación son, tanto del investigador, como los sujetos investigados, entiéndase, con ello, que asumen un papel activo, en la construcción de conocimiento.

Dado lo anterior se asumirá como entrada metodológica histórica una postura hermenéutica; de tal manera, que se hagan 
procesos interpretativos, al recuperar la experiencia de los participantes como maestros y estudiantes en la escuela rural. Buscando con ello, ser fieles a lo vivido y narrado por ello, en tanto que los investigadores se posicionan como garantes de las comprensiones y significados de las voces de los actores con la mayor transparencia posible, tal como lo señala Marín (9), al referirse a la interpretación en la investigación cualitativa, resaltando que "supone buscar significados, entender lo que ocurre más allá de lo observable, inferir y detectar interacciones complejas y latentes, para construir teorías válidas en las ciencias sociales y humanas." (9, p. 100).

La investigación asumió referentes epistemológicos sistémico complejos. Dado que en la contemporaneidad, surgieron comprensiones emergentes, en contraposición a las formas de explicar los fenómenos sociales, educativos, que primaron en la modernidad; más específicamente a las perspectivas empírico analíticas, que de acuerdo con los planeamientos de Habermas (10), al referirse a los intereses constitutivos del conocimiento, organizaron una forma de comprensión humana, desde lo técnico, lo comprobable, los resultados, lo observable; entre otros; aspectos que posiblemente eran aplicables a los fenómenos matemáticos, económicos y biológicos; sin embargo no sucedía lo mismo para tomarlos como referentes que permitieran entender lo humano.

En el paradigma sistémico complejo autores como Bertalanffy (11) aportando el concepto de sistema y causalidad circular, Foerster (12), a partir de la cibernética de segundo orden y Morín (13), con su propuesta sobre los sistemas autopoyeticos, entre otros; además de cuestionar la forma mecanicista del conocer; ofrecen principios, que replantean la perspectiva del investigador en tanto que hace parte del proceso mismo de la investigación; al hacerse la transición entre sistemas observadores a sistemas observantes. Por ejemplo, al identificar los procesos interacciónales como focos centrales de la investigación, para apreciar la complejidad humana en relación con los contextos, sus historias de vida, el micro y macro sistemas en los que se está inmerso (14).

Con base en los objetivos específicos de la investigación, se establecieron 3 categorías deductivas.

Primera, hace referencia a las creencias de los maestros sobre la violencia escolar.

Segunda cuenta de los tipos de violencia que circulan en dicho contexto.

Tercera relacionada con los efectos de la violencia escolar en la salud mental de maestros y estudiantes.

Participantes: 6 escuelas rurales del departamento de Córdoba, territorio históricamente considerado afectado por el conflicto armado colombiano, se seleccionaron dieciocho maestros. Dado que la investigación fue de carácter cualitativo, se definieron como aspectos representativos de la cantidad de participantes, lo que (15) proponen como selección basada en criterios; para este caso, consistieron en ser miembro de la comunidad educativa por más de 5 años en calidad de docente, ser egresado de licenciatura en pregrado o tener estudios relacionados con educación, tener a cargo cursos de básica primaria y querer participar en la investigación.

\section{RESULTADOS}

Con base en las narrativas de los participantes, se organizó la información por cada categoría en matrices, para ello se asignó a cada participante un código alfanumérico, y poder identificar la procedencia de los testimonios. A los maestros se les asigno la letra (m) y con el número de participante, por ejemplo, m3, corresponde al maestro número 3 . A continuación se presentan, por cada objetivo específico, extractos de las narrativas de los participantes, como ejemplos de las categorías deductivas y las emergentes.

Se procedió a realizar la selección de la información, de acuerdo con los criterios aportados por Cisterna (16), es decir, la pertinencia y la relevancia; el primero para tomar en cuenta lo que se relaciona con la temática de la investigación y el segundo da cuenta de lo que se devela por recurrencia y asertividad en relación con los objetivos propuestos. Para el análisis de los resultados se diseñaron matrices de doble entrada, lo cual permitió realizar el análisis de contenido para llegar a las interpretaciones buscadas.

Con respecto al primer objetivo específico, que buscaba reconocer las creencias de los maestros sobre la violencia escolar coinciden en su totalidad los 18 participantes, en que es cualquier forma de agresión física, verbal o psicológica la cual se puede presentar entre todos los miembros de la comunidad educativa y no permite la sana convivencia, se basa en falta de valores tanto en la familia como en la sociedad en general. Así mismo emergieron otras subcategorías; que permiten comprender de manera más específica las creencias.

En la tabla 1 se presentan las emergentes, que configuran las creencias sobre la violencia en la escuela. Se establecen de mayor a menor presentación las tendencias, de acuerdo con las intervenciones de los participantes. Para ello, se agruparon los textos surgiendo las siguientes subcategorías.

Tabla 1: Categorías emergentes sobre Creencias de violencia escolar.

\begin{tabular}{lc}
\hline \hline \multicolumn{1}{c}{ Categoría deductiva: Creencias sobre violencia } \\
\hline \multicolumn{1}{c}{ Sub Categorías Emergentes } & Tendencia presentación. \\
\hline $\begin{array}{l}\text { Violencia como forma de proteger los } \\
\text { derechos personales ante la comunidad } \\
\text { escolar. }\end{array}$ & 17 \\
\hline $\begin{array}{l}\text { Dificultad en manejo dimensión emocional } \\
\text { en estudiante con fuerte influencia familiar. }\end{array}$ & 14 \\
\hline $\begin{array}{l}\text { Contexto de violencia naturalizado en la } \\
\text { comunidad. }\end{array}$ & 14 \\
\hline $\begin{array}{l}\text { Currículo centrado en contenidos y no en } \\
\text { formación del ser. }\end{array}$ & 10 \\
\hline \hline
\end{tabular}


A continuación, se presentan extractos de narrativas de los participantes en los que se ilustran, a manera de ejemplo cada una de las subcategorías emergentes.

En relación con la subcategoría violencia como opción para proteger los derechos personales ante la comunidad escolar, se encontraron las siguientes intervenciones ". ...La mayoría de los niños piensan que cuando actúan de forma agresiva se están haciendo respetar, están imponiendo su autoridad; porque en nuestro contexto se da mucho eso, que se dice: que el que más fuerte habla y el que más vocabulario obsceno usa es el que más se le escucha, y en algunos casos dentro de la sociedad misma se da que los adultos se pelean y dicen a mí nadie me mete en el bolsillo". (m9).

En la subcategoría dificultad en el manejo de emociones en el estudiante con una fuerte influencia familiar, se encontraron las intervenciones que dan cuenta de ello; "Son aquellas emociones no bien dirigidas u orientadas por un ser, una persona hacia el otro. Cuando nosotros no sabemos manejar nuestras emociones pues nos metemos en conflicto, yo pienso que es generado más que todo por una falta de valores, actualmente pues las familias están desagregadas, no hay un patrón de conducta, en que lo haya no es el mejor referente para los estudiantes, para los niños, entonces yo pienso que desde allí se está generando la violencia escolar y los conflictos que se dan a causa de esto (m17).

Otro texto relacionado con la misma subcategoría se evidencia así, "Teniendo en cuenta que también no viven con los padres, viven con abuelos u otras terceras personas y que muchas veces estas personas no les brindan el apoyo, el amor que el muchacho necesita de acuerdo a la etapa en que se encuentre, consiguen de pronto amistades, las amistades que de pronto si les brinden ese apoyo que en casa deben darle, no tienen un buen modelo para saber expresar sus emociones, vienen de comunidades vulnerables, vienen de pronto buscado un poquito de amor acá a las escuelas". (m8)

Con referencia a la subcategoría del Contexto de violencia social naturalizado en la comunidad, se presentan algunos ejemplos detectados, al respecto un participante precisó que "Seguramente el estudiante quiere expresarse, o de pronto manifestar situaciones que se presentan en su entorno familiar. Esta es una zona que está influenciada por grupos al margen de la ley y que ellos son la autoridad en la región y es posible que eso se pueda manifestar en los hogares y se refleje en la actuación de los jóvenes acá, niños, adolescentes" (m16).

Otro texto que lo evidencia se refiere a "Los niños cuentan que los maltratan los jefes de los grupos al margen de la ley a ellos o a sus padres y esto también los afecta mucho. De pronto ellos ven mucho eso o también el reflejo dentro del mismo hogar, de los papas que son agresivos, se maltratan, se agreden física o verbalmente, porque la presión aquí es muy fuerte, para que no se lleven a los hijos; entonces esos son los reflejos de ellos a veces, la rebeldía de pronto empieza en la casa y la reflejan acá en el aula de clase” (m14).

De otra parte, se ilustra la subcategoría sobre el currículo basado en contenidos y no en la formación del ser; en la siguiente narrativa “....es que las escuelas se están enfocando por las directrices ministeriales, ósea al fortalecimiento de algunas áreas y no a su crecimiento de talentos, entonces un niño con talentos reprimidos todavía va ser un poco más violento, por no poder mostrar sus capacidades, lo que tiene el para mostrarle al mundo". (m11). Otro maestro también manifiesta que "nos presionan para que los niños aprendan sobre matemáticas, lenguaje, y las áreas, pero sobre la convivencia no queda tiempo y menos en sus aptitudes" (m2).
Para dar cuenta del segundo objetivo específico, el cual buscaba identificar los tipos de violencia en el contexto escolar; se encontraron subcategorías relacionadas con el lugar en el cual se presentan tanto dentro del aula, como las que circulan en otros escenarios de la escuela. También se estructuraron por tipos de participantes, a partir de las interacciones entre docentes con estudiantes, padres de familia con estudiantes y entre compañeros de clase. Estas mismas se categorizaron de mayor a menor grado de violencia. En la tabla 2, se representan las tendencias encontradas sobre tipos de violencia y escenarios escolares en las que se presentan.

Tabla 2: Categorías emergentes sobre Tipos de violencia y escenarios escolares en interacción entre estudiantes.

Categoría deductiva: Tipos de violencia y escenarios escolares

\begin{tabular}{cc}
\hline $\begin{array}{c}\text { Sub Categorías Emergentes en interacciones } \\
\text { entre estudiantes }\end{array}$ & $\begin{array}{c}\text { Tendencia } \\
\text { presentación. }\end{array}$ \\
\hline Violencia verbal y psicológica dentro del aula & 15
\end{tabular}

Violencia verbal y psicológica en los baños y 11 sus alrededores.

\begin{tabular}{lc}
\hline $\begin{array}{l}\text { Violencia verbal y psicológica en los espacios } \\
\text { para el recreo. }\end{array}$ & 8 \\
\hline Violencia física. & 1 \\
\hline \multicolumn{2}{c}{ Elaboración propia. Tendencias de presentación emergentes para } \\
categoría 2. Interacciones entre estudiantes
\end{tabular}

Con respecto a las violencias dentro del aula entre estudiantes, se evidenció que están relacionadas con lo verbal, como formas de expresión que surgen cuando se presentan conflictos por cuestiones personales, familiares o posibles hurtos en pertenencias de estudiantes; provocan discusiones a través de vocabulario soez, apodos, gestos, burlas. En menor escala se da la violencia física y el ciber acoso, "matoneo" entre compañeros y porte de armas blancas. Para dar cuenta de lo anterior se presentarán los siguientes textos. "Como le dije, el pegar es poco, sin embargo, colocar sobre nombres, tirarle las cosas al compañero al piso, botarle las cosas al compañero, reírse del compañero, esas son pequeñas acciones más frecuentes, pero que se consideran violencia" (m4).

También en el siguiente relato se evidencia cuando el participante comenta que "se dicen malas palabras, se ponen apodos, entonces cuando ellos se maltratan de esa manera poniéndose apodos o diciendo malas palabras y responden con agresividad, ya se van a los golpes, se pegan, en algunas ocasiones inician con un juego, un juego como de manoteo, de manos, como si estuvieran jugando a la lucha libre o algo así, y como alguno de ellos salga maltratado, terminan dándose golpes de verdad y eso genera violencia, también, afortunadamente no se da tanto; solo en casos muy extremos " (m12).

En relación con la presencia de violencia entre estudiantes en otros escenarios de la institución escolar diferentes al aula; se encuentran narrativas como "Fuera del aula cuando están jugando, como no les pongan reglas al juego o ponen reglas y son incumplidas entonces se maltratan de manera verbal" (m16). También refieren los participantes, en los baños y sus alrededores se intensifican dichos episodios; como se observa en el siguiente texto del maestro "...las peleas se dan más en los baños, porque algunos llegan a atropellar al otro, le hacen maldades, por ejemplo, le echan agua y generan ese tipo de situaciones. En el baño se da una violencia digamos que psicológica, podemos decirlo, se escriben cosas bastante grotescas, en las paredes de los sanitarios y las niñas, entre niñas se colocan un poco de mensajes. Por ejemplo, las niñas como forma de manifestarse, de pronto no se dan 
golpes, pero generalmente si se escriben cosas grotescas ahí en el baño entonces toca mandar a limpiar los baños." (m18).

Sumado a esto, se da una mayor tendencia a emplear agresiones verbales y psicológicas en espacios para el recreo de los estudiantes; tal es el caso representado en el siguiente texto "...Lo que más se da es la burla entre los compañeros, se ponen sobre nombres, apodos, alias. Porque hasta ahora no hemos tenido información de que hay violencia de pronto por la red" (m13). Se encontró también que pocas veces se detecta violencia física, como se aprecia en el siguiente texto “...Se golpean más bien poquito, se agreden verbalmente, más agresiones verbales, con malas palabras, sobrenombres; nunca hemos tenido casos de niños que son agredidos físicamente o con armas blancas" (m10).

Por otro lado, en las interacciones entre docentes y estudiantes se encontraron las siguientes tendencias, las cuales se representan en la siguiente tabla.

\section{Tabla 3: Categorías emergentes sobre Tipos de violencia interacción docentes estudiantes.}

\section{Categoría deductiva: Tipos de violencia y escenarios escolares}

\begin{tabular}{lc}
\hline \hline \multicolumn{1}{c}{$\begin{array}{c}\text { Sub Categorías Emergentes en interacciones } \\
\text { docentes estudiantes }\end{array}$} & $\begin{array}{c}\text { Tendencia } \\
\text { presentación. }\end{array}$ \\
\hline $\begin{array}{l}\text { Violencia psicológica dentro del aula asociada } \\
\text { al uso de rótulos a partir del déficit académico } \\
\text { del estudiante. }\end{array}$ & 18 \\
\hline $\begin{array}{l}\text { Violencia verbal dentro del aula, por presiones } \\
\text { personales del docente ante niños } \\
\text { indisciplinados. }\end{array}$ & 6 \\
\hline \multicolumn{2}{c}{ Elaboración propia. Tendencias de presentación emergentes para categoría 2. } \\
Interacciones entre docentes y estudiantes.
\end{tabular}

Sobre los tipos de violencia en las relaciones pedagógicas entre estudiantes y profesores "uno ve compañeros que les ponen sobre nombres a los estudiantes, apodos por su poca responsabilidad o rendimiento en las materias; como docentes podemos generar nuestras prácticas y nuestra forma de actuar en el aula, expresiones que aparentemente pueden ser las más adecuadas o chistosas, pero que violentan un poca la mente de los muchachos". (m5). Otro participante evidencia que la dimensión personal y familiar del maestro, es un factor importante; como se manifiesta en el siguiente extracto "El maestro también tiene muchas cargas en su vida, lo económico, las dudas o con sus parejas; tiene que cumplir en la institución y se descarga con los estudiantes, con el niño más inquieto o que no cumple y se desespera y lo grita, usa términos ofensivos como sobrenombres" (m.15)

En relación con el tercer objetivo específico que buscaba identificar los efectos de la violencia escolar en la salud mental de estudiantes y maestros, se evidencio que existe una preocupación de los maestros por la creciente situación de violencia que se está presentando cada vez más y con episodios más agresivos, sobre todo entre los estudiantes; generándose ambientes altamente conflictivos y problemas en la autoestima; reflejada en timidez, dejarse manipular para ser aceptado y manifestación de ansiedad ante la presentación de exámenes y cumplimiento de tareas escolares. Ello se apreció tanto en los alumnos que abusan verbal, psicológica o físicamente de sus compañeros como de los que son víctimas de ello. En la siguiente tabla se presentan las subcategorías emergentes que dan cuenta de este objetivo de investigación.

\section{Tabla 4: Emergentes sobre efectos en salud mental} estudiantes ante la violencia escolar.

Categoría deductiva: salud mental estudiantes

\begin{tabular}{lc}
\hline \hline \multicolumn{1}{c}{$\begin{array}{c}\text { Sub Categorías Emergentes en salud mental } \\
\text { estudiantes ante la violencia escolar }\end{array}$} & $\begin{array}{c}\text { Tendencia } \\
\text { presentación. }\end{array}$ \\
\hline $\begin{array}{l}\text { Aumento de episodios y carga de agresividad } \\
\text { en episodios de violencia entre estudiantes. }\end{array}$ & 18 \\
\hline Dificultades en autoestima. & 17 \\
\hline $\begin{array}{l}\text { Relaciones conflictivas generadoras de } \\
\text { ambientes altamente estresantes. }\end{array}$ & 17 \\
\hline \hline & $\begin{array}{l}\text { Elaboración propia. Tendencias de presentación emergentes para categoría 3. Efectos en salud mental } \\
\text { estudiantes ante la violencia escolar. }\end{array}$
\end{tabular}

En los siguientes textos se ejemplifican los hallazgos sobre cada emergente. Un maestro manifestó que "Desde un punto más que todo social, se traduce aquí en la institución porque ellos expresan o es la forma de protegerse creo que emocional e ideológicamente, de la violencia social y económica que viven en los hogares, se traduce acá, cada vez más en la violencia que ellos expresan con el matoneo hacia los compañeros, es una forma de expresarse, algunos se vuelven víctimas y otros abusadores, produciéndose problemas en la autoestima" (m7).

Además, otro participante, manifiesta que “...ellos tienen distintas formas de expresar como un camino para resolver sus frustraciones, al ser maltratados en sus familias o no tener lo que quieren, no saben decir lo que sienten y actúan agresivamente, cada vez son más violentos, algunos viven muy estresados, casi que asustados" ( $\mathrm{m} 1)$. Otro ejemplo se evidencia cuando un maestro señala que "es el mal trato, el mal vocabulario, esa interacción que se tiene con otra persona, que afecta la autoestima en los niños más que todo y los vuelve adultos con problemas ya sea inseguros o violentos, el problema es que se dejan manejar por sus otros compañeros, si comparamos cada año, los maestros hemos observado que es más grave a medida que pasa el tiempo. (m3).

De otra parte, los docentes encuentran que los estados de ansiedad al percibirse en situación de minusvalía e incertidumbre, se incrementa especialmente cuando se presentan situaciones producto del conflicto armado en las zonas donde están ubicadas las escuelas. Manifiestan necesitar apoyo para manejar estas vivencias, dado que sienten presión por parte de los padres de familia, los niños y en general de la comunidad, para que sean un soporte y apoyo en dichos eventos.

\section{Tabla 5: Emergentes sobre efectos en salud mental de} maestros ante la violencia escolar.

\begin{tabular}{lc}
\hline \hline \multicolumn{2}{c}{ Categoría deductiva: salud mental estudiantes } \\
\hline \hline $\begin{array}{c}\text { Sub Categoría Emergentes en salud mental de } \\
\text { maestros ante la violencia escolar }\end{array}$ & $\begin{array}{c}\text { Tendencia } \\
\text { presentación. }\end{array}$ \\
\hline $\begin{array}{c}\text { Estados de ansiedad por situaciones de } \\
\text { conflicto armado, que afectan la convivencia }\end{array}$ & 14 \\
en la escuela. & \\
\hline \hline & Elaboración propia. Tendencias de presentación emergentes para categoría 3. \\
Efectos en salud mental de los maestros ante la violencia escolar
\end{tabular}

$\mathrm{Al}$ respecto un participante, comento que "los profesores, nos vemos muy afectados por lo que les pasa a estos niños, sobre todo cuando hay sucesos de violencia en el pueblo... uno está muy nervioso, no duerme, la pensadera de lo que va a pasar lo mata a uno y no sabe cómo ayudar a los niños (m4). Otro maestro, señaló que "Esperan mucho de uno, 
los papas le confían a uno sus hijos, aquí en el pueblo los profesores todavía somos respetados y a mí, casi que se me sale el corazón por si les pasa algo cuando hay tomas de grupos al margen de la ley; yo a veces veo que hay compañeros como que ni quieren venir a trabajar, están muy preocupados, estresados... es difícil.” (m11).

\section{DISCUSIÓN}

Con respecto a las creencias sobre violencia escolar de acuerdo con Llinares (6), quien postula la presencia de intenciones, argumentos y acciones en la configuración de estas. Se puede afirmar que las intenciones de los actos violentos están enmarcadas en la necesidad de los estudiantes por hacer valer sus derechos y ganar respeto ante la comunidad escolar, lo cual se evidencia en sus comportamientos intolerantes y con dificultad en el manejo de la dimensión emocional tanto en ellos como en sus familias.

El argumento que da sustento a ello se estructura a partir de la naturalización en diversos sistemas sociales principalmente en las familias y comunidad; sobre la validez de implementar actos violentos, como alternativa para resolver conflictos y resolver problemas. No obstante, Lo anterior permite comprender que se requiere un abordaje sistémico a nivel educativo, que va más allá del aula, dado que lo presentado en la escuela se convierte en un isomorfismo (11) de lo que se replica en una mayor escala tanto en las familias como en la comunidad en general, sobre los actos violentos y las formas en que se presentan.

Dado el valor que tiene para la comunidad el papel de la escuela en las zonas rurales Colombianas; esta se convierte como un entorno protector (16) para la salud mental de la red familia escuela y comunidad; al posicionarse como un escenario de reflexión y acción entre los diferentes participantes de la comunidad para generar estrategias que favorezcan la preservación de los derechos de los niños, niñas y adolescentes; favoreciendo la comunicación con los adultos; en aras del desarrollo de sus capacidades, recursos personales y sus proyectos de vida.

Se aprecia con preocupación el aumento tanto en frecuencia, como en carga de agresividad de los episodios de violencia en la escuela; lo cual convoca de manera urgente a realizar procesos de intervención como estrategia preventiva; para ello es necesario trabajar desde las creencias de los diferentes actores de la comunidad escolar, logrando con ello construir nuevos referentes que le confieran el valor de acertado y válido a las nuevas prácticas que construyan para hacer valer sus derechos y empoderarse como sujetos valiosos en sus argumentos, estilos y acciones ante la comunidad.

\section{CONCLUSIONES}

Considerar la escuela como un entorno protector a partir de una pedagogía Resiliente.
La importancia de reconocer las experiencias significativas de los maestros como buenas prácticas, para a partir de ello, construir alternativas que vayan más allá del aula y se puedan transformar en innovación de la institución educativa. En este sentido se ve la necesidad que los maestros sistematicen sus experiencias, para construir relatos pedagógicos contextualizados con estrategias efectivas, para prevenir violencia escolar y preservar la salud mental de los miembros de la institución educativa.

$\mathrm{Al}$ abordar las creencias se trasciende el nivel de la acción, el cual es importante, pero no suficiente ante la complejidad de la situación encontrada sobre la violencia escolar; Por ello bajo este referente también se trasforman sistémicamente las intenciones y los argumentos que tienen las personas como marcos lógicos para proceder y que consideran válidos. En este sentido, a partir de dicha movilización en el sistema de creencias; se pueden generar cambios contextualizados, pertinentes y duraderos en el tiempo.

Para construir las estrategias de intervención pedagógica, se debe partir del reconocimiento de los recursos personales de los estudiantes y maestros, de las familias y de la comunidad; que han construido en sus experiencias ante las situaciones de violencia tanto dentro de la escuela como fuera de ella; para que conjuntamente desde sus historias y creencias emerjan herramientas acordes a sus realidades. No se trata entonces que las intervenciones educativas se orienten a decir a la comunidad lo que se debe hacer para construir escenarios sin violencia; sino más bien, configurar con ellos lo que es más procedente a sus realidades.

La pedagogía Resiliente se puede establecer como pilar central para la intervención; la cual se fundamenta en una mirada sistémica y apreciativa del otro; con base en la identificación de las practicas acertadas tato en la familia como en la escuela que han favorecido la construcción de escenarios sin violencia ante situaciones adversas; Por ejemplo se han de diseñar portafolios de estrategias pedagógicas de afrontamiento que han sido útiles en la historia de la comunidad, para el manejo de situaciones que afectan negativamente la autoestima en los estudiantes, generan ansiedad, estrés e incertidumbre relacional; como efectos en la salud mental de maestros y estudiantes frente a la violencia escolar.

Conflicto de Interés: Los investigadores declaran no tener ningún conflicto de interés en la realización del estudio.

\section{REFERENCIAS}

1. Comisión Económica para América Latina de las Naciones Unidas., CEPAL. Violencia social. 2017; Disponible en: https://www.cepal.org/es/temas/violencia-social. Accessed 03/02/, 2020.

2. Castañeda AE, Gómez. JA, Vargas GR. Pedagogía resiliente. Tunja (Colombia): Ediciones USTA.; 2019.

3. Ministerio de Salud. Ley 1616 de 2003. 2003. Disponible en: https://www.minsalud.gov.co/sites/rid/Lists/BibliotecaDigit 
al/RIDE/DE/DIJ/ley-1616-del-21-de-enero-2013.pdf. Accessed 03/02/, 2020.C

4. Vargas Rodríguez GR, Castañeda Cantillo AE, Gómez Arévalo JA. Violencia y escuela en escenarios rurales: Informe final de investigación. Universidad Santo Tomás Abierta y a Distancia. 2018. Disponible en: https://search.ebscohost.com/login.aspx?direct=true $\& \mathrm{db}=\mathrm{ir}$ 01372a\&AN=rin.11634.14681\&lang=es\&site=eds-live Accessed 03/02/, 2020.

5. Llinares-Ciscar S. El conocimiento y las creencias de los profesores de matemáticas y la innovación educativa. Revista Investigación en la Escuela 1990; 11:61-69. Disponible en: http://revistas.usal.es/index.php/02125374/article/view/3558 Accessed 03/02/, 2020.

6. Eljach S. Violencia escolar en América Latina y el Caribe Superficie y fondo. . Fondo de las Naciones Unidas para la Infancia. UNICEF 2011. Disponible en: https://www.unicef.org/costarica/docs/cr_pub_Violencia_es colar_America_Latina_y_Caribe.pdf Accessed 03/02/, 2020.

7. López N, Sandoval I. Métodos y técnicas de investigación cuantitativa y cualitativa. Disponible en: http://recursos.udgvirtual.udg.mx/biblioteca/bitstream/2005 0101/1103/1/Metodos_y_tecnicas_de_investigacion_cuanti tativa_y_cualitativa.pdf Accessed 03/02/, 2020.

8. Marín Gallego JD. La investigación en educación y pedagogía. Sus fundamentos epistemológicos y metodológicos. Bogotá (Colombia): Ediciones USTA; 2012.

9. Habermas J. Conocimiento e interés, Selección de los apartados IV-VII. Lección inaugural tenida el 28 del 6 de 1965 en la Universidad de Frankfurt, con el título original "Erkenntnis und Interesse", y publicada por primera vez en diciembre de ese mismo año en Merkur. Universidad de Frankfur 1965.

10. Bertalanffy LV. Teoría General de los Sistemas. Fundamentos, desarrollos y aplicaciones. Madrid (España): Fondo de Cultura Económica; 1956.

11. Foerster HV. Las Semillas de la Cibernética. Barcelona (España): Gedisa; 1991.

12. Morín E. El método. Naturaleza de la naturaleza. 6th ed. Madrid (España): Ediciones Cátedra; 1993.

13. Castañeda AE. Evaluación del aprendizaje. Una perspectiva sistémica. Bogotá (Colombia): Ediciones USTA.; 2017.

14. Goetz JP, LeCompte MD. Etnografía y diseño cualitativo en investigación cualitativa. Madrid (España): Ediciones Morata S.A.; 2010.

15. Cisterna Cabrera F. Categorización y triangulación como procesos de validación del conocimiento en investigación cualitativa. Universidad del Bío Bío 2005;14(1):61-71. Disponible

en: http://www.redalyc.org/articulo.oa?id=29900107 Accessed 03/02/, 2020.

16. Instituto Colombiano de Bienestar familiar., ICBF. Construyendo Juntos Entornos Protectores. 2019; Available at: https://www.icbf.gov.co/programas-y-estrategias/ninez$\mathrm{y}$-adolescencia/construyendo-juntos-entornos-protectores. Accessed 03/02/, 2020.

17. Ministerio de Educación (2014). ABECÉ, Sobre la Salud Mental, sus Trastornos y Estigma. Disponible en:https://www.minsalud.gov.co/sites/rid/Lists/BibliotecaD igital/RIDE/VS/PP/abc-salud-mental.pdf 